\title{
La PET y PET-CT en la estadificación y tratamiento del cáncer de pulmón no microcítico
}

\author{
M. Provencio ${ }^{1}$, A. Sánchez ${ }^{1}$, C. González², F. Valcárcel ${ }^{3}$, A. Varela ${ }^{4}$, A. Maldonado ${ }^{5}$, \\ J. González-Alenda ${ }^{5}$, J. Moradiellos ${ }^{4}$, J. L. Chamorro
}

\section{Resumen}

La tomografía por emisión de positrones con 2-( $\left.{ }^{18} \mathrm{~F}\right)$-fluoro-2-desoxi-D-glucosa (PET-FDG) es una técnica metabólica de imagen. La PET-FDG es más precisa que el CT en la valoración de la afectación mediastínica en los pacientes con carcinoma de pulmón no microcítico, con un alto valor predictivo negativo. Puede detectar metástasis ocultas en el $11 \%$ de los pacientes, si bien, la etiología de las captaciones solitarias extratorácicas debe ser confirmada. La PET-FDG, teóricamente, puede influir en el volumen de planificación de la radioterapia, sobre todo en los pacientes con atelectasia. La cuantificación de la actividad metabólica mediante la PET-FDG está influenciada por el tamaño de la lesión, los niveles de glucosa, el tiempo que transcurre desde la inyección del isótopo. Se necesitan más ensayos clínicos para la estandarización de los métodos de realización del PET, su uso como factor pronóstico y para la valoración de la respuesta al tratamiento.

Palabras clave: Tomografía de emisión de positrones. PET-FDG. Cáncer de pulmón no microcítico.

Oncología, 2007; 30 (3):100-112

Servicio de Oncología Médica

${ }^{2}$ Radiodiagnóstico

${ }^{3}$ Radioterapia

${ }^{4}$ Cirugía Torácica

${ }^{5}$ Medicina Nuclear

Hospital Universitario Clínica Puerta de Hierro. Madrid (España)

${ }^{6}$ Centro PET Recoletas La Milagrosa 


\section{Summary}

Positron emission tomography with (18F)2-fluoro-2-deoxy-D-glucose (PET-FDG) is an imaging metabolic technique. PET-FDG is more precise than CT for the evaluation of mediastinal affectation of patients with non-small cell lung cancer, offering a high negative predictive value. It can detect occult metastases in $11 \%$ of the patients, although the etiology of the extra-thoracic isolated receptions needs confirmation. Theoretically, PET-FDG can influence the planning volume of radiotherapy, mainly of patients with atelectasis. The quantification of the metabolic activity by means of PET-FDG is influenced by the size of the lesion, the glucose levels, and the time elapsed since the isotope injection. More clinical assays are needed to standardize PET execution procedures, its use as a prognostic factor and the evaluation of treatment response.

Key words: Positron emission tomography. PET-FDG. Non-small cell lung cancer.

\section{Introducción}

El tratamiento de los pacientes con cáncer de pulmón no microcítico (CPNM) se establece según el estadio tumoral. La clasificación TNM proporciona la información más precisa sobre la resecabilidad y pronóstico del tumor ${ }^{1}$. La correcta estadificación es esencial para indicar el tratamiento adecuado. La tomografía por emisión de positrones (PET) es un método complementario a la tomografía axial computerizada $(\mathrm{CT})$ en la determinación de la extensión del CPNM ${ }^{2}$. La PET con 2-( $\left.{ }^{18} \mathrm{~F}\right)$-fluoro-2-desoxi-Dglucosa (PET-FDG) se basa en el elevado metabolismo de la glucosa en el interior de las células tumorales en comparación con las células sanas, que se produce como resultado de un incremento en la expresión de las proteínas transportadoras de la glucosa como la GLUT1 y de la hexoquinasa (una enzima que fosforila la glucosa). El $\left[{ }^{18} \mathrm{~F}\right] \mathrm{FDG}$ intracelular es el sustrato básico del PET. La imagen de la PET-FDG se realiza por la rápida capacidad para minimizar la inhibición competitiva de la captación de FDG por la glucosa.

A continuación, estudiaremos el papel del PET, sólo o asociado al CT en diferentes situaciones clínicas: estadificación tumoral, pronóstico y en respuesta al tratamiento.

\section{Estadificación tumoral}

El estudio de la extensión del tumor primario pulmonar se realiza mediante $\mathrm{CT}$ y, en ocasiones se complementa con resonancia magnética en determinadas situaciones clínicas como tumores del sulcus superior o para establecer la relación con estructuras vasculares como los grandes vasos o el corazón. En contraste con la CT, que aporta una imagen anatómica, la PET-FDG se basa en la actividad biológica de las células tumorales. Las células tumorales del cáncer de pulmón muestran una alta captación y utilización de la glucosa comparadas con las células del tejido pulmonar o linfático ${ }^{3}$. Sin embargo, esta técnica tiene limitaciones en su capacidad para la localización anatómica de las lesiones. Algunos procesos no tumorales como granulomas o enfermedades inflamatorias, y las infecciones pueden mostrar captaciones positivas en PET-FDG. Por otra parte, la capacidad de resolución del PET es de 4-6 mm, dependiendo de la intensidad de la captación del isótopo por las células normales ${ }^{4}$. Todo ello, hace que en tumores con tamaño inferior a $2 \mathrm{~cm}$ la PET-FDG sea de poca utilidad con un alto porcentaje de falsos negativos $(45 \%)^{5}$. Por lo tanto, debido a su limitada resolución espacial no tiene un papel específico en la estadificación del tumor primario. 
Algunos estudios indican que la PET-FDG puede ser más útil en la evaluación del derrame pleural. La toracocentesis puede no ser diagnóstica en un 30\%$40 \%$ de los pacientes que tienen derrame pleural tumoral. En un estudio, a 35 pacientes con cáncer de pulmón y derrame pleural objetivado en CT, se les realizó PET-FDG ${ }^{6}$. La sensibilidad, especificidad y exactitud diagnóstica del PET fueron 89\%, 94\% y $91 \%$, respectivamente. En otro estudio, la sensibilidad, especificidad y exactitud fueron $95 \%, 67 \%$, y $92 \%$, respectivamente ${ }^{7}$.

Numerosos estudios han evaluado el valor del CT en la estadificación del mediastino ${ }^{8-10}$. En la mayoría de ellos, el principal criterio para la valoración de la afectación mediastínica era la presencia de adenopatías mayores de $10 \mathrm{~mm}$. En un metaanálisis, Toloza $^{11}$ y cols. analizaron diversos estudios que comparaban el CT con mediastinoscopia o con cirugía. La sensibilidad de la CT fue 0.57 (95\% CI, 0,49 - 0,66) y la especificidad de 0.82 (95\% CI, 0.77 0.86). El valor predictivo positivo (VPP) fue de 0.56 y el valor predictivo negativo (VPN) de 0.83 . En conclusión, la CT muestra un elevado número de resultados falsos positivos. Además, estudios realizados entre 1980 y 1988 mostraron los mismos re- sultados que en la última década, a pesar de la mejora de la resolución de la CT . Por lo tanto, el estudio con CT es insatisfactorio para la determinación de la afectación mediastínica (N2 y N3), especialmente desde que su afectación se considera un punto crítico para la consideración de enfoques quirúrgicos. Sin embargo, debido a su alta resolución espacial, la CT de tórax se considera una prueba diagnóstica útil para la confirmación histológica del mediastino, ya que permite la selección de las adenopatías para la punción-aspiración o para la mediastinoscopia ${ }^{12}$.

En el metaanálisis más reciente ${ }^{13}$, la sensibilidad de la PET-FDG para la detección de la afectación mediastínica variaba entre el $66 \%$ y el $100 \%$ (Fig. 1). La sensibilidad global estimada fue del $83 \%$ (95\%, CI 77 - 87). La especificidad variaba entre el $81 \%$ y el $100 \%$, con una especificidad global estimada del 92\% (95\% CI, 89 - 95). La sensibilidad y la especificidad de la CT para la detección de la afectación mediastínica variaba entre el $20 \%$ y el $81 \%$, y entre el $44 \%$ y el $100 \%$, respectivamente. La sensibilidad y especificidad estimada fue del $59 \%$ (95\% CI, 50 - 67) y del 78\% (95\% CI, 70 $84)$, respectivamente.
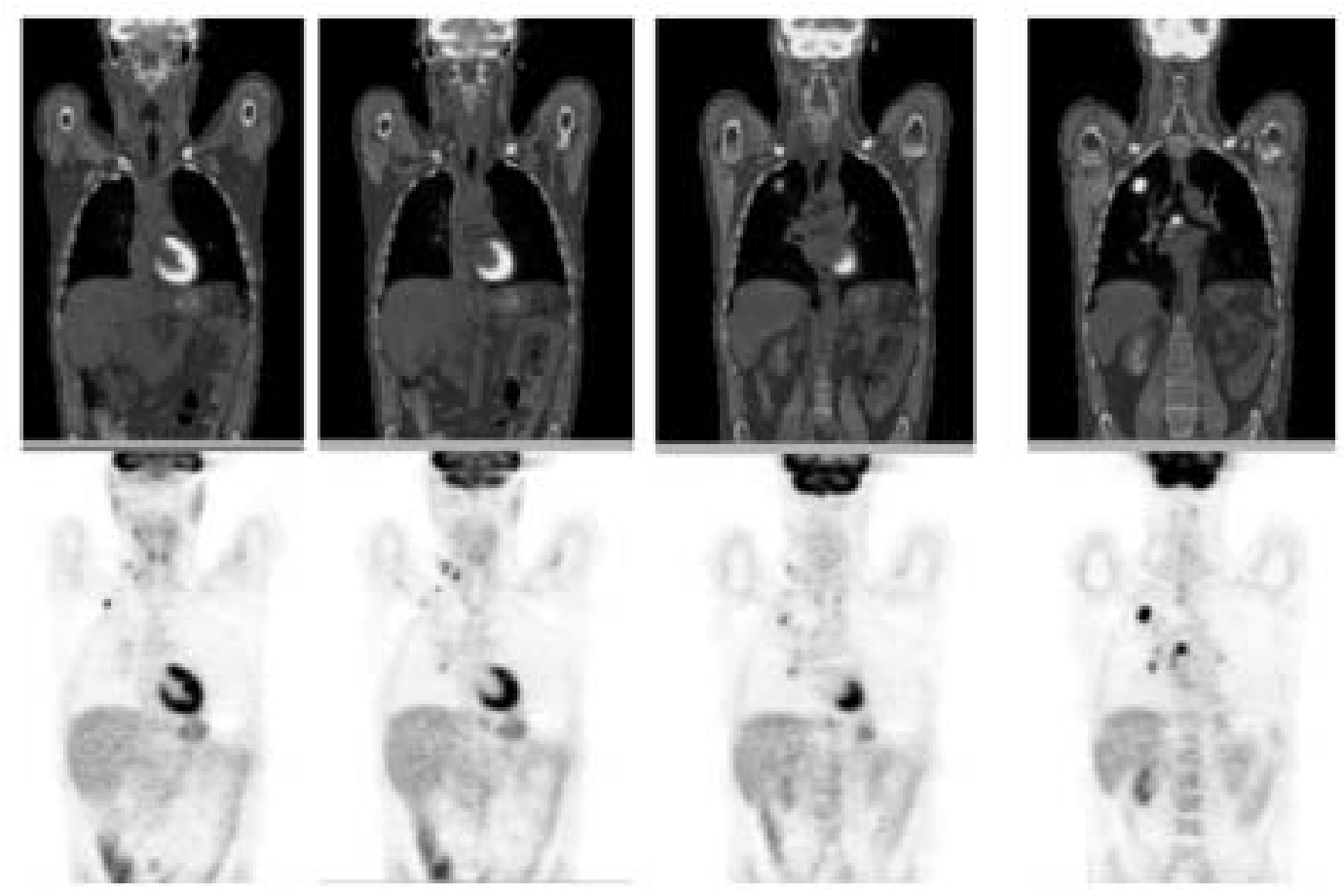

Figura 1. Lesión maligna en LSD. Adenopatías en región cervical derecha, fosa supraclavicular, región axilar ipsilateral así como a nivel subcarinal e hilios pulmonares. 
Comparando los resultados de la PET-FDG con los del examen histológico y citológico se encontró que: la sensibilidad fue del 0.84 (95\% CI, 0.79 $0.89)$ y la especificidad del 0.89 (95\% CI, 0.83 0.93). El VPP fue de 0.79 (95\% CI, $0.40-1.00)$ y el VPN fue de 0.93 (95\% CI, 0.75 - 1.00). Por lo tanto, en el caso de un PET positivo, la probabilidad de un resultado falso positivo fue del $21 \%$, lo que puede influenciar de manera significativa la elección del tratamiento (quirúrgico o no). Se recomienda confirmar anatomopatológicamente un resultado positivo del PET en el mediastino, en el caso de enfermedad no metastásica ${ }^{14,15}$. Además, el PET es incapaz de distinguir focos metastásicos menores de $4 \mathrm{~mm}$. En todo caso, se recomienda la confirmación patológica de la afectación mediastínica diagnosticada por PET-FDG ${ }^{16}$ teniendo en cuenta además que el número de falsos positivos puede estar entre el 20 y $25 \%$.

Sin embargo, cuando la CT muestra afectación mediastínica patológica, la PET-FDG es más sensible pero menos específica ${ }^{17}$ que la CT . Debido a las limitaciones de su resolución espacial, a menudo no es posible distinguir el tumor primario o la afectación ganglionar hiliar de la afectación mediastínica. Esto se valoró en un reciente estudio con 400 pacientes, donde se observó que era más probable no detectar la afectación N2 en los ganglios subaórticos y subcarinales ${ }^{18}$.

En el ensayo PLUS ${ }^{19}, 188$ pacientes con sospecha de CPNM fueron aleatorizados a realizar un estudio de extensión convencional con/sin PET, previo a la valoración quirúrgica de la enfermedad. En el grupo de pacientes en los que se realizó PET-FDG, hubo un 51\% (95\% CI, 32-80\%; p:0.003) de reducción de toracotomías innecesarias y un $8 \%$ de afectación metastásica no conocida. La adición del PET al estudio de extensión convencional podría prevenir cirugías innecesarias en el $20 \%$ de los pacientes con sospecha de CPNM. Sin embargo, el estudio presenta un importante número de problemas metodológicos, incluyendo una pobre estadificación en el grupo control, con un pequeño número de mediastinoscopias $\mathrm{y}$, un escaso uso de CT de nueva generación, sólo realizado en el $55 \%$ de los pacientes ${ }^{20}$. Otros dos grandes ensayos no demuestran una disminución del número de toracotomías en los pacientes en los que se incluyó la PET-FDG en la estadificación preoperatoria ${ }^{21,22}$.

Además, la PET-FDG muestra limitaciones en la valoración de algunos tipos de tumores de metabolismo singular como son el carcinoide y el carcinoma bronquioalveolar. Recientes estudios asocian la expresión de transportadores de glucosa de membrana con el grado de diferenciación y el subtipo histológico ${ }^{23}$.

En resumen ${ }^{24}$, los estudios con PET-FDG presentan un elevado VPN (94\%) en la estadificación del mediastino. Por lo tanto, en los pacientes sin afectación mediastínica en PET se podría llevar a cabo la cirugía directamente.

La mediastinoscopia, tal como afirma Rusch, continua siendo el "gold standard" en la estadificación prequirúrgica del mediastino, pero en el futuro podría ser sustituida por la PET-CT y la punción con aguja fina (PAAF) guiada por endoscopia ${ }^{25}$. Por ahora, la PET y la CT son técnicas complementarias y ambas deben interpretarse en el contexto de los resultados de la otra.

\section{Lesiones metastásicas}

A pesar de la mayor sensibilidad, especificidad y exactitud diagnóstica de la PET-FDG en la detección de la extensión metastásica, no conocemos bien su papel y si podría reemplazar a las pruebas radiológicas convencionales, debido a que en la mayoría de los estudios éste se realizó como una técnica adicional $^{26}$.

La incidencia de metástasis ocultas diagnosticadas mediante PET-FDG varió entre el 9 y el $24 \%$ 2,27,28. El número de las metástasis detectadas por PET-FDG aumentó conforme aumentaba la estadificación del paciente, desde el $8 \%$ en los estadios I, al $18 \%$ en el estadio II, y hasta al $24 \%$ en el estadio III ${ }^{29}$ (Tabla I).

TABLA I

Valor de la PET-FDG en la detección de metástasis no conocidas

\begin{tabular}{|lcc|}
\hline Estudio & $N^{o}$ pacientes & \% detección metastasis \\
\hline Bury $^{37}$ & 109 & 6 \\
MacManus $^{29}$ & 167 & 19 \\
Marom $^{33}$ & 100 & 9 \\
Pieterman $^{4}$ & 102 & 11 \\
Stroobants $^{44}$ & 144 & 5 \\
Valk $^{27}$ & 99 & 11 \\
Weder $^{28}$ & 100 & 15 \\
\hline
\end{tabular}



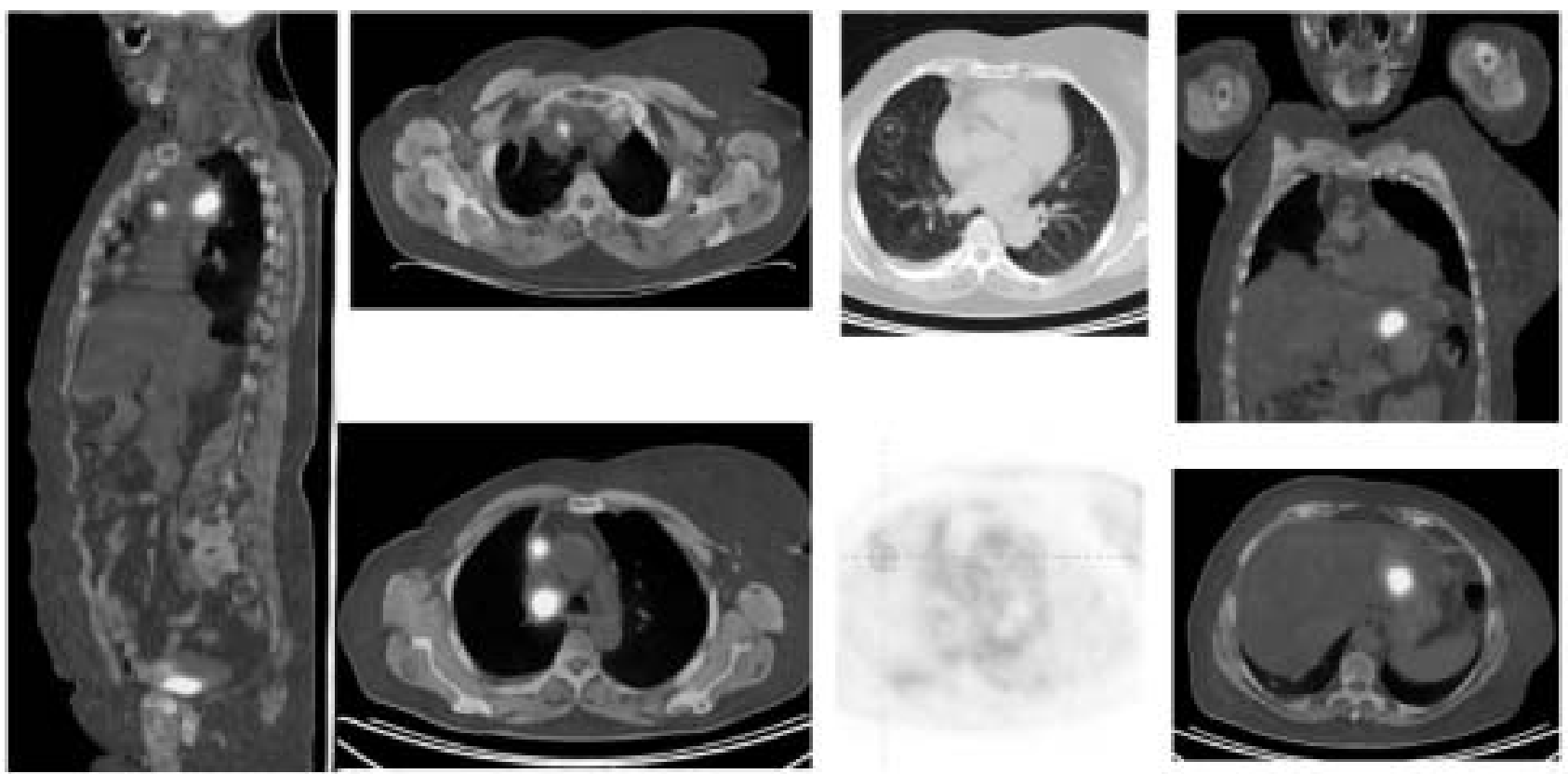

Figura 2. Lesiones malignas en hemitórax derecho y mediastino. Metástasis hepática.

Cuando la PET-FDG muestra una única captación extratorácica se presenta una situación clínica problemática debido a que la PET-FDG no es específica de malignidad. Lardinois en un excelente estudio $^{30}$ muestra que el PET-CT descubre lesiones únicas extrapulmonares en el $21 \%$ de los pacientes con CPNM (Fig. 2). En la mitad de los casos, las lesiones no se correspondían con metástasis, sino que correspondían con otros tumores malignos, como se había observado en anteriores estudios ${ }^{31} \mathrm{o}$, con lesiones benignas. Por tanto, la etiología de las captaciones solitarias extrapulmonares de la PET-FDG deben ser confirmadas histológicamente, más cuando la actitud terapéutica puede variar por estos hallazgos. Se debe prestar especial atención a las captaciones solitarias en el bazo, axila, canal espinal, tracto gastrointestinal y páncreas.

En casi el $10 \%$ de los pacientes con CPNM, las glándulas suprarrenales se encuentran aumentadas de tamaño al diagnóstico en el estudio de CT. Aproximadamente dos tercios de estas lesiones suprarrenales van a ser benignas. Incluso, la resonancia magnética muestra un elevado número de falsos positivos $^{32}$. La PET-FDG puede ser útil en esta situación, ya que su alta sensibilidad (95\%-100\%) y especificidad (80\%-100\%) puede permitir una reducción del número de biopsias suprarrenales ${ }^{33-36}$.
La PET-FDG no es útil para la detección de metástasis cerebrales, dado que la corteza cerebral presenta un elevado consumo de glucosa. Su sensibilidad es baja $(60 \%)$ debido a la alta afinidad de la glucosa por el tejido cerebral normal.

En la actualidad, la afectación ósea generalmente se evalúa mediante gammagrafía ósea con ${ }^{99 \mathrm{~m}} \mathrm{TC}-\mathrm{MDP}$, que tiene una buena sensibilidad $(90 \%)$ pero una baja especificidad $(60 \%)$ por el elevado número de falsos positivos debidos a la captación del radionúclido en cualquier área de elevada actividad ósea. La PET-FDG tiene una sensibilidad similar (90\%) pero una mayor especificidad (>98\%) y exactitud (>96\%), y un menor número de falsos positivos en los casos de procesos degenerativos, inflamatorios, y post-traumáticos comparados con la gammagrafía, por lo que se puede considerar superior en la detección de la afectación ósea metastásica ${ }^{37-39}$.

\section{Impacto en el estadificación y tratamiento de los pacientes}

Diferentes estudios han analizado los cambios que se producen en la estadificación e incluso en el tratamiento de los pacientes con la utilización de la PET-FDG . 
TABLA II

PET-FDG en la estadificación y cambio de tratamiento de los pacientes

\begin{tabular}{|lccc|}
\hline Estudio & $\begin{array}{c}N^{o} \\
\text { pacientes }\end{array}$ & $\begin{array}{c}\text { Cambios de } \\
\text { estadio (\%) }\end{array}$ & $\begin{array}{c}\text { Impacto en el } \\
\text { tratamiento (\%) }\end{array}$ \\
\hline Bury $^{37}$ & 109 & 34 & 25 \\
Hicks $^{41}$ & 153 & 43 & 35 \\
Hoekstra $^{71}$ & 57 & 30 & 19 \\
Pieterman $^{4}$ & 102 & 62 & - \\
Schmucking $^{42}$ & 63 & - & 52 \\
\hline
\end{tabular}

Un reciente estudio publicado en Oncología en nuestro país, el PET modificaba el tratamiento inicialmente previsto en el $30 \%$ de lo pacientes estudiados con cáncer de pulmón ${ }^{40}$ pasando de una cirugía radical a quimioterapia o quimiorradioterapia. En la Tabla II resumimos alguno de los estudios publicados, el cambio en el estadio varía entre el 27 al $60 \%$ y el impacto sobre clara modificación en el tratamiento entre el 25 y el $50 \%$.

\section{Valor pronóstico al diagnóstico}

Se han estudiado varios métodos de imagen basados en el metabolismo tumoral ${ }^{43}$. La captación de $\left[{ }^{18} \mathrm{~F}\right] \mathrm{FDG}$ por las células tumorales del CPNM se asocia con el crecimiento y la proliferación celular. La medida de la captación in vivo mediante PETFDG usando el SUV (standarised uptake value) se ha investigado como una posible variable de agresividad y factor pronóstico ${ }^{44}, 45$. El SUV es un valor semicuantitativo y se ha asociado con factores de proliferación celular como Ki-67 y el antígeno nuclear de proliferación celular ${ }^{46,47}$. En series retrospectivsas $^{48-53}$ de pacientes con CPNM se ha sugerido que el SUV en el tumor primario pulmonar en el momento del diagnóstico es predictivo de control de la enfermedad y de supervivencia. El punto del corte del SUV en los análisis univariantes de los diferentes estudios es muy variable, desde 5 a $7,10,15$ o incluso 20.

El SUV proporciona información pronóstica independiente, a parte del estadio y tamaño tumoral, estadio y estado general o estadio sólo. En un estudio reciente ${ }^{54}$, el SUV del tumor primario fue el factor pronóstico más importante entre los pacientes trata- dos con cirugía curativa o radioterapia. Los pacientes con valores bajos de SUV (<5.0) presentaron significativamente una mejor supervivencia libre de enfermedad, que los pacientes con valores elevados (>5.0). Sin embargo, usando el mismo punto de corte de 5, el valor del SUV para la afectación linfática regional no fue un factor significativo en la supervivencia libre de enfermedad. El análisis multivariante mostró que el SUV del tumor primitivo fue un factor pronóstico significativo para la supervivencia global y la supervivencia libre de enfermedad.

Por otra parte, hay varios factores que pueden influir cuantitativamente en las medidas de la captación de la glucosa por las células tumorales ${ }^{55}$. Así, los carcinomas escamosos parecen presentar un SUV superior al adenocarcinoma ${ }^{56}$. El tamaño tumoral tiene una influencia significativa en el valor del SUV. Existe una marcada subestimación de la captación del $\left[{ }^{18} \mathrm{~F}\right] \mathrm{FDG}$ en los tumores de hasta 3-4 $\mathrm{cm}$. Sólo cuando el diámetro de la lesión es sobre 4 veces el tamaño de la resolución espacial del CT , las diferencias entre la concentración del pico medido de actividad y la concentración de la actividad real es menor del 5\%. Igualmente, la captación puede ser subestimada en los tumores con necrosis central, con baja captación de $\left[{ }^{18} \mathrm{~F}\right] \mathrm{FDG}$, incluso si el diámetro de todo el tumor es mayor de $4 \mathrm{~cm}$. Otros factores que pueden influir en la captación son: niveles de glucosa en sangre, más baja con el incremento de los niveles de glucosa, al competir esta por el transportador; y también el periodo de tiempo tras la inyección del radiofármaco, ya que existe un aumento de la captación de $\left[{ }^{18} \mathrm{~F}\right] \mathrm{FDG}$ del $50 \%$ entre los 40 y 90 minutos tras la inyección ${ }^{57}$.

Weber realiza una comparación interindividual realizando dos pruebas en el mismo paciente y esperando un periodo de 4-6 semanas tras la finalización del tratamiento. Esta estrategia reduce de manera significativa el número de factores que pueden influir en la señal del $\left[{ }^{18} \mathrm{~F}\right] \mathrm{FDG}$. La precisión del la PET-FDG para la medida de los cambios en la glucosa tumoral es considerablemente mejor que la ofrece para la cuantificación del uso de la glucosa tumoral en unidades absolutas.

Se necesitan más estudios para la estandarización de los métodos de realización de la PET-FDG y de la medida del SUV, para definir los mecanismos celulares subyacentes, y la validación del valor pronóstico basal de la captación de $\left[{ }^{18} \mathrm{~F}\right] \mathrm{FDG}$. 


\section{Respuesta al tratamiento}

El sistema de regresión gradual de la captación de la PET-FDG sugiere ser un factor pronóstico significativo en los pacientes con CPNM localmente avanzado tras el tratamiento neoadyuvante ${ }^{58}$. En otros tumores, como en el linfoma ${ }^{59,60}$, cáncer esofágico ${ }^{61}$, ${ }^{62}$, cáncer de estómago ${ }^{63}$, y osteosarcoma ${ }^{64}$ una disminución en la captación de $\left[{ }^{18} \mathrm{~F}\right] \mathrm{FDG}$ ha demostrado ser lo más exacto para la predicción de la regresión histopatológica.

Dado que el $\left[{ }^{18} \mathrm{~F}\right] \mathrm{FDG}$ se acumula preferentemente en la células tumorales viables y no en el tejido necrótico, un cambio en la captación de $\left[{ }^{18} \mathrm{~F}\right] \mathrm{FDG}$ en el PET pudiera ser una buena variable para la monitorización de la respuesta, y, en teoría, podría ser capaz de objetivar una respuesta precoz. Recientemente, el activo grupo de UCLA ha publicado un estudio que ilustra la base racional de la utilización de glucosa y el efecto de gefitinib sobre un panel de líneas celulares con un espectro de sensibilidad a los inhibidores de EGFR quinasa ${ }^{65}$. Encuentran una clara disminución de la captación de glucosa muy precozmente en aquellas líneas celulares sensibles a gefitinib, que precede en el tiempo a otros cambios celulares y a la apotosis. Con las limitaciones que los estudios sobre líneas celulares que los mismos autores reconocen, éste aporta varios aspectos de mucho interés. Primero que la investigación de la respuesta mediante MicroPET puede hacerse desde niveles básicos de cultivos celulares, y por otra parte aporta datos importantes para la evaluación de PET en predicción de respuesta a inhibidores de la EGFR quinasa. Sin duda un camino muy atractivo que sería deseable continúe explorándose en el futuro.

El papel de la PET-FDG en la reestadificación de los pacientes con CPNM tras tratamiento neoadyuvante con quimioradioterapia no ha sido completamente evaluado (Fig. 3). Hay cinco estudios prospectivos $^{66-70}$ que evalúan el valor de la PETFDG en la monitorización de la respuesta a la quimioterapia o radioterapia en pacientes con CPNM. Estos estudios difieren sustancialmente en el tamaño, criterios de selección de pacientes, procedimientos de imagen e interpretación, pero todos ellos indicaron un posible papel de la PET-FDG en la determinación de la respuesta al tratamiento. Indican que la PET-FDG es útil en la monitorización del tratamiento neoadyuvante quimioradioterápico en pacientes con CPNM. La rápida identificación de los pacientes no respondedores mediante la PET-FDG podría ser beneficioso al reducir significativamente los efectos secundarios y el coste de un tratamiento
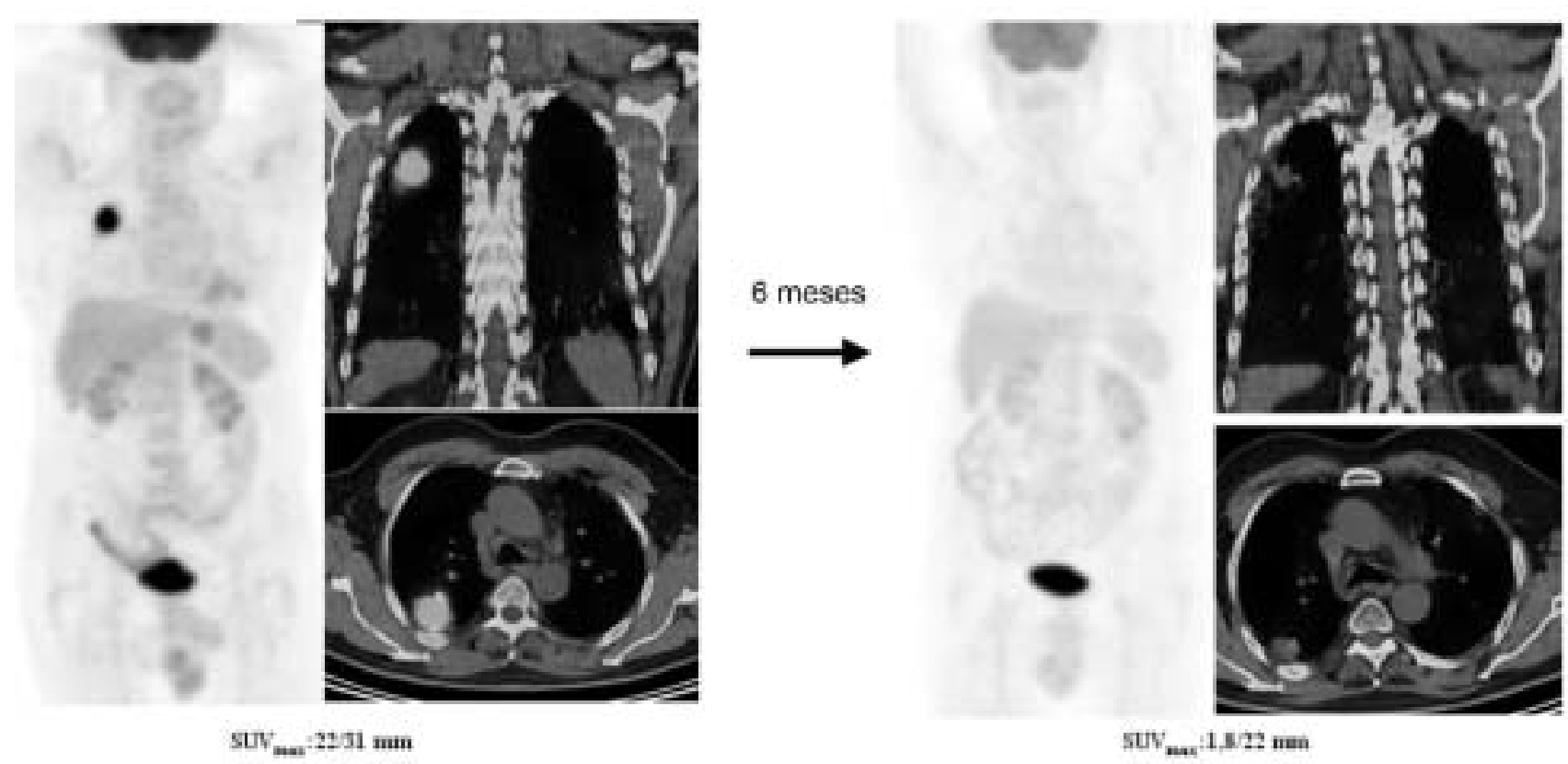

Figura 3. Disminución de tamaño y marcada disminución de actividad metabólica de la lesión neoplásica conocida en segmento II de pulmón derecho. 
no eficaz. En todos estos estudios, la PET-FDG tuvo un alto VPN en la valoración de la respuesta.

Hoekstra $^{71}$ y cols. realizaron un análisis comparativo de los métodos del PET y, la exactitud diagnóstica de la medida de la respuesta precoz usando el PET respecto a la supervivencia en los pacientes en estadio IIIA-N2 que han recibido quimioterapia neoadyuvante. La PET-FDG se realizó antes de la quimioterapia y tras el primer y tercer ciclo. La CT se realizó antes y después del tratamiento. Tras el primer ciclo de quimioterapia, la presencia de captación focal en el mediastino no tuvo valor predictivo respecto a la supervivencia $(\mathrm{p}=0.74)$. Pero al final del tratamiento, la determinación del mediastino por PET-FDG predijo la supervivencia de los pacientes (hazard ratio (HR), 2.33; 95\% CI, 1.04 5.22; $\mathrm{p}=0,04)$ en comparación con la CT (HR, 1.87; 95\% CI, 0.81 - 4.30; p=.14). Los autores estudiaron el consumo de glucosa (metabolic rate of glucose $\left.\left[M R_{\text {glu }}\right]\right)$ y la glucosa residual tras el tratamiento $\left(\mathrm{MR}_{\mathrm{glu}}\right)$, que fue el mejor factor pronóstico (HR, 1.95; 95\% CI, 1.28 - 2.97, p=0.002). La sensibilidad y especificidad de la PET-FDG tras la quimioterapia neoadyuvante en pacientes $\mathrm{N} 2$ fue $50 \%$ (95\% IC, $19 \%$ - 81\%) y $71 \%$ (95\% CI, $42 \%-92 \%)$ respectivamente, y el correspondiente VPP y VPP fue $66 \%(95 \%$ CI, $21 \%$ - $86 \%$ ) y $67 \%(95 \%$ CI, $38 \%-88 \%)$. Estos datos indican que la captación residual durante o después del tratamiento podría ser un buen marcador pronóstico. Se pueden considerar algunos parámetros relacionados con $\mathrm{MR}_{\mathrm{glu}}$ para obtener una estratificación pronóstica: cambio fraccional, niveles basales o residuales del metabolismo de la glucosa.

El valor de la PET-FDG en la monitorización de la respuesta es complejo de determinar debido a la heterogeneicidad de los datos publicados con respecto a los métodos aplicados de $\mathrm{PET}^{72}$, y a los diferentes objetivos clínicos en los estudios. En general, la determinación del intervalo óptimo de su realización desde la administración del tratamiento quimioterápico neoadyuvante no está aclarado y la evidencia en los estudios de neoadyuvancia es todavía demasiado limitada.

\section{Planificación de la radioterapia}

En teoría la PET-FDG puede influir el volumen planificado del tratamiento con radioterapia y pro- porcionar una reducción de la dosis sobre tejido sano. Además, el fracaso local confirmado mediante biopsia ocurre hasta en el $83 \%$ de los estadios III, indicando la necesidad de mejorar el control local, a pesar de las altas dosis de radiación administradas.

Numerosos estudios evalúan la información que aporta la PET-FDG en la planificación de la radioterapia. En un estudio de viabilidad con varios tumores sólidos, la información proporcionada por la PET-FDG cambiaba el volumen tumoral en 22/39 (56\%) si la información de la imagen metabólica era usada en la planificación ${ }^{73}$. En los pacientes con CPNM estadio N2-N3, el uso de la PET-FDG en la planificación del tratamiento con radioterapia, reducía la exposición a la radiación del esófago y del pulmón, y de este modo permitía una significativa escalada de la dosis respetando los límites de dosis en los tejidos $\operatorname{sanos}^{74}$. Además, se observó la presencia de variación interobservador y este factor contribuyó a las diferencias en la determinación de los límites tumorales ${ }^{75,76,77}$.

Vanuystel y cols. estudiaron una comparación teórica del tamaño de los volúmenes tumorales (gross tumor volumes, GTVs) definidos por CT, y mediante ambos PET-FDG y CT . Los hallazgos de la PET-FDG alteraron los volúmenes teóricos en 45 de 73 pacientes $(62 \%) .{ }^{78}$ Bradley, en un reciente estudio prospectivo ${ }^{79}$, analizó cómo los hallazgos de la PET-FDG alteraban el estadio TNM en 8 de 26 pacientes $(31 \%)$. De los 24 pacientes que fueron planificados mediante el método de la radioterapia conformacional tridimensional, la PET-FDG claramente alteró el volumen de radiación en 14 (58\%). La PETFDG ayudó a delimitar el tumor dentro de las áreas de atelectasia en tres pacientes, reduciendo el GTV de cada uno. La adición de la PET-FDG no modificó el estadio asignado a ninguno de los pacientes. Por otra parte, incrementó el GTV en 11 pacientes.

Deniaud-Alexandre ${ }^{80}$ y cols., en un estudio retrospectivo, confirmaron que el PET-TAC tiene impacto en la planificación del tratamiento y el manejo del CPNM. En este estudio, con un total de 101 pacientes, la PET-FDG identificó metástasis ocultas en el 8 pacientes (8\%), y el GTV disminuyó, con la fusión de las imágenes del PET-CT , en 21 pacientes y aumentó en 24 pacientes. La reducción del GTV fue $>25 \%$ en 7 pacientes $(7 \%)$ debido a que la fusión de imágenes del PET-CT redujo el GTV en 6 
pacientes ( 3 de ellos con atelectasia) y la afectación mediastínica en 1 paciente. El aumento del GTV fue $>25 \%$ en 14 pacientes (14\%) debido a un incremento en el GTV en 11 pacientes (4 de ellos con atelectasia) y la detección de afectación mediastínica no conocida en 3 pacientes. El análisis multivariante mostró que el tumor con atelectasia fue el único factor independiente que resultó tener un efecto significativo en la modificación del tamaño del GTV mediante PET-CT.

El uso del PET en la simulación aumentará la precisión de acuerdo con el GTV. Pero, está por definir qué es lo que constituye una alteración significativa en la delimitación de los contornos del tumor. Pensamos que las variaciones interobservador son un factor considerable. De otra parte, el GTV puede sobreestimarse por inflamación crónica, neumonía y reacción granulomatosa peritumoral.

Los datos previos ofrecen suficiente apoyo para la realización de estudios prospectivos, longitudinales multiinstitucionales para la validación de estos resultados y la evaluación del control tumoral, supervivencia, y modificaciones por el uso del PET.

La combinación de la CT torácica y el PET se ha estudiado recientemente ${ }^{81}$. Los primeros estudios fueron prometedores. El PET-CT podría minimizar la dosis de radiación y el PET en el tórax sería igualmente útil que el PET corporal.

Aquino y cols.$^{82}$, demostraron que los resultados mejoraban cuando las imágenes del PET-CT se correlacionaban con las imágenes del CT, y mostraron que los resultados eran mejores cuando el PET-CT y el CT se fusionaban digitalmente ${ }^{83}$. El registro de imágenes del PET y de la CT y la integración del PET-CT proporcionan una adecuada fusión de imágenes e incluso se espera que una mejor estadificación del mediastino.

En un estudio de Cerfolio, el PET-CT proporcionaba mayor precisión en el estudio de la afectación ganglionar ${ }^{84}$.

Antoch y cols.$^{85}$, y Lardionois y cols, concluyeron que el PET-CT mejoraba la precisión diagnóstica de la estadificación del CPNM. En contraste, Vansteenkiste $^{86}$ y Magnani no encontraron diferencias significativas en la precisión con las imágenes de fusión digital del PET-CT, ni tampoco al analizar la estadificación ganglionar ni las distintas cadenas ganglionares linfáticas.
Halpern y cols. ${ }^{87}$, publicaron recientemente que la precisión de las imágenes del PET-CT era significativamente mejor que con el PET $(\mathrm{p}<0.05)$. Las imágenes de fusión obtenidas separadamente del PET y del CT fueron útiles en el 68\% de los pacientes.

En nuestro país, Pozo-Rodriguez ${ }^{88}$ y cols, encontraron que la CT helicoidal y el PET mostraban similares resultados en la estadificación mediastínica, ambas pruebas eran condicionalmente dependientes y proporcionaban información complementaria, y su valor diagnóstico residía principalmente en los resultados negativos. En este estudio, la sensibilidad y especificidad de la CT fue 0.86 (95\% CI, $0.70-$ $0.93)$ y 0.67 (95\% CI, $0.56-0.75)$, y para el PET de $0.94(95 \%$ CI, $0.81-0.98)$ y $0.59(95 \%, 0.49$ 0.68). Para la CT helicoidal y el PET combinados en paralelo fue de 0.97 (95\% CI; 0.84 - 0.99) y de 0.44 (95\% CI, 0.34 - 0.53).

En un estudio con diferentes tipos de tumores, las imágenes del PET-CT se compararon en un análisis por separado con las imágenes de la CT y del PET. La combinación de ambos mejoraba la caracterización de las lesiones equívocas como benignas en un $10 \%$ de los sitios y como malignas en un $5 \%{ }^{89}$.

Una grave limitación es que la mayoría de los estudios sobre PET-CT no son comparativos con PET sólo, además de las graves dificultades metodológicas para hacer comparaciones, puesto que si hay algo común es la baja calidad de los estudios publicados.

Así, en España, la Agencia de Evaluación de Tecnologías Sanitarias ha realizado una excelente revisión sistemática y un meta-análisis sobre las indicaciones del PET-CT ${ }^{90}$. De 209 artículos seleccionados inicialmente, sólo 16 se pudieron emplear para el metaanálisis. De estos, 12 eran estudios prospectivos y 4 retrospectivos, y existía gran variedad de tipos de PET-CT, con diferentes tumores estudiados y varias indicaciones del procedimiento. En el estudio concreto de la estadificación del CPNM, el PETCT aportó una sensibilidad agregada de 0.85 (IC $95 \%, 0.74-0.92)$ y una especificidad agregada de 0.84 (IC95\%, 0,70 - 0.93). Para la re-estadificación tumoral, la sensibilidad de la PET-CT fue de 0.89 (IC 95\%, 0.84 - 0.94) y la especificidad de 0.87 (IC $95 \%, 0.78$ - 0.93). Los autores concluyeron que esta técnica combinada es una tecnología útil en la estadificación y re-estadificación tumoral, porque au- 
menta el nivel de confianza en el diagnóstico al disminuir el número de lesiones equívocas o no concluyentes. En conclusión, el PET-CT ${ }^{91}$ parece mejor que el CT para el estadificación del CPNM y proporciona una mayor precisión y especificidad en la estadificación ganglionar.

Aún así, como en otros meta-análisis realizados sobre el PET que hemos ido comentando con anterioridad, llama la atención la disparidad de los estudios, la utilización de los mismos pacientes para distintas publicaciones, el carácter retrospectivo de muchas de ellas, el escaso nivel de calidad de gran parte de los estudios realizados y publicados, en algunos no se conoce ni el género de los pacientes, mezcla de histologías, ..., así como el posible sesgo de utilizar sólo estudios publicados en lengua inglesa que, por lo general, son aquellos que han proporcionado resultados positivos.

La cuestión no es baladí, son numerosos los recursos económicos destinados a estas exploraciones, además de las repercusiones diagnósticas y terapéuticas que pueden generar, sin contar la presión ambiental por parte de pacientes reclamando pruebas o conclusiones de las mismas que no siempre pueden ser efectuadas con los datos que disponemos. Por todo ello hay acuerdo global en reclamar que los estudios sobre test diagnósticos, y, en concreto sobre PET o PET-CT, cumplan con criterios de calidad y control similares a otros procedimientos de investigación médica a los que estamos acostumbrados en el desarrollo de la Oncología.

\section{Agradecimientos}

Realizado gracias al Convenio de colaboración del "Instituto Salud Carlos III y "Agencia Laín Entralgo" dentro del programa de estabilización e intensificación de la actividad investigadora del Sistema Nacional de Salud.

\section{Bibliografía}

1. Sobin LH, Wiyyekind C eds.TNM classification of malignant tumours.6th ed. New York: John Wiley \& Sons, 2002.

2. Vansteenkiste J, Fischer BM; Dooms C, Mortensen J. Positron-emission tomography in prognostic and therapeutic assesment of lung cancer: systematic review. Lancet Oncol 2004; 5: 531-40.

3. Nolop KB, Rodees CG, Brudin LH, et al. Glucose utilization in vivo by human pulmonary neoplasms. Cancer 1987; 60: 2682-2689.

4. Pieterman RM, van Putten JW, Meuzelaar JJ, et al. Preoperative staging of non-small-cell lung cancer with positron-emission tomography. N Engl J Med 2000; 343: 254-261.

5. Port JL, Andrade RS, Levin MA, et al. Positron emission tomographic scanning in the diagnosisi and staging of nonsmall cell lung cancer $2 \mathrm{~cm}$ in size or less. J Thorac Cardiovasc Surg 2005; 130: 1611-1615.

6. Gupta NC, Rogers JS, Graeber GM, et al. Clinical role of F-18 fluorodeoxyglucose positron emission tomography imaging in patients with lung cancer and suspected malignat pleural effusion. Chest 2002; 122:1918-1924.

7. Erasmus JJ, McAdams HP, Rossi SE, et al. FDG PET of pleural effusions in patients with non-small cell lung cancer. AJR Am J Roentgenol 2000; 175: 245-249.

8. Kramer H, Groen HJM. Current concepts in the mediastinal lymph node staging in suspected lung cancer: Comparison of positron emission tomography with F-18 fluorodeoxyglucose and mediastinoscopy. Ann Thorac Surg 2003; 75: 231-235.

9. Dales RE; Stark RM, Raman S. Computed tomography to stage lung cancer. Approaching a controversy using metaanalysis. Am Rev Respir Dis 1990; 141: 1096-1101.

10. Dwamena BA, Sonnad SS, Angobaldo JO, et al. Metastases from non-small cell lung cancer: Mediastinal staging in the 1990s-metaanlytic comparison of PET and CT. Radiology 1999; 213: 530-536.

11. Toloza EM, Harole L, Mc Crory DC. Noninvasive staging of non-small cell lung cancer: a review of the current evidence. Chest 2003; 123(1 suppl): 147S-156S.

12. Silvestri GA, Tanoue LT, Margalis L, Barker J, Detterbeck $\mathrm{F}$. The noninvasive satging of non-small cell lung cancer: the gidelines. Chest 2003; 123 (1 sppl): 147S-156S.

13. Birim O, Kappetein AP, Stijnen T, Bogers JJC. Metaanalysis of positron emission tomographic and computed tomographic imaging in detecting mediastinal lymph node metastases in non-smallcell lung cancer. Ann Thorac Surg 2005; 79:375-81.

14. Detterbeck FC, Falen S, Rivera MP, Halle JS, Socinski MA. Seeking a home for a PET, part 2: defining the appropiate place for positron emission tomography imaging in the staging of patinets with suspected lung cancer. Chest 2004; 125: 2300-2308. 
15. Spiro SG, Porter JC. Lung cancer-where are today? $\mathrm{Cu}-$ rrent advances in staging and nonsurgical treatment. Am J Resir Crit Care Med 2002; 166:1166-1196.

16. Pfister DG, Johson DH, Azzoli CG, et al. American Society of Clinical Oncology treatment of unresectable nonsmall-cell lung cancer guideline: update 2003. J Clin Oncol 2004; 22: 330-53.

17. Gould MK, Kuscher WG, Rydzak CE, et al. Test performance of positron emission tomography and computed tomography for mediastinal staging in patients with nonsmall lung cancer: a meta-analysis. Ann Intern Med 2003; 139: 879-892.

18. Cerfolio RJ, Ojha B, Bryant AS, et al. The role of FDGPET scan in staging patients with non-small cell carcinoma. Am Thorac Surg 2003; 76:861-866.

19. Van Tinteren H, Hoekstra OS, Smit EF, et al. Effectiveness of positron emisión tomography in the preoperative assessment of patients with suspected non-small cell lung cancer: the PLUS multicentre randomised trial. Lancet 2002; 359: 1388-1393.

20. Passlick B. Mediastinal satging (take home messages). Lung Cancer 2004; (suppl 2) : s85-s87.

21. Viney RC, Boyer MJ, King MT, et al. Randomized controlled trial of the role of positron emission tomography in the management of stage I and II non-small-cell lung cancer. J Clin Oncol 2004; 22: 2357-62.

22. Herder GJ, et al Tradiotional versus up-front 18FDG PET staging of non-small cell lung cancer: a Ducth co-operative randomized study. (abstract). Proc Am Soc Clin Oncol 2004; 23:615a.

23. de Geus-Oei LF, Krieeken JH, Aliredjo RP et al. Lung Cancer 2006; oct 12 [Epub ahead of print]

24. Groen HJM, Sleijfer DT, de Vries EG. Positron emission tomography computerized tomography, and endoscopic ultrasound with needle aspiration for lung cancer. American Society of Clinical Oncology. Educational Book. 2005; 579-585.

25. Rusch VW. Mediastinoscopy: An endangered species? J Clin Oncol 2005; 23: 8283-8285.

26. Ukena D, Hellwing D. Value of FDGPET in the managenement of NSCLC. Lung cancer 2004; 45 (suppl 2): s75-s78

27. Valk PE, Pounds TR, Hopkins DM, et al. Staging nonsmall cell lung cancer by whole-body positron emission tomographic imaging. Ann Thorac Surg 1995; 60: 1573-81.

28. Weder W, Schmid RA, Bruchlaus $\mathrm{H}$ et al. Detection of extrathoracic metastases by positron emission tomography in lung cancer. The Ann Thorac Surg 1998; 66: 886-892.

29. MacManus MP, Hicks RJ; Matthews J, et al. High rate of detection of unsuspected distant metastasesby PET in apparent stage III non-small cell lung cancer: implications for radical radiation therapy. Int J Radiat Oncol Biol Phys 2001; 50: 287-293.

30. Lardinois D, Weder W, Roudas M, et al. Etiology of solitary extrapulmonar positron emisión tomography and computed tomography findings in patients with lung cancer. J Clin Oncol 2005; 23: 6846-6853.
31. Choi JY, Lee KY, Kwon OJ, et al. Improved detection of second primary cancer using integrated $18 \mathrm{~F}$ fluorodeoxyglucose positron emission tomography and computed tomography for initial tumor staging. J Clin Oncol 2005; 23: 7654-7659.

32. Burt M, Heelan RT, Coit D, et al. Prospective evaluation of unilateral adrenal masses in patients with operable nonsmall cell lung cancer: Impact of magnetic resonace imaging. J Thorac Cardiovasc Surg 1994; 107: 584-588.

33. Marom EM, Mac Adams HP, Erasmus JJ, et al. Staging non-small cell lung cancer with whole-body PET. Radiology $1999 ; 212 ; 803-809$.

34. Jana S, Zhang T, Milstein DM, et al. FDG-PET and CT characterization of adrenal lesions in cancer patients. Eur J Nucl Med Mol Imaging 2006; 33: 29-35.

35. Kumar R, Xiu Y, Yu J, et al. 18F-FDG PET in evaluation of adrenal lesions of patients with lung cancer. J Nucl Med 2004; 45: 2058-2062.

36. Metser U, Miller E, Lerman H, Lievshitz G, Avital S, Even-Sapir E. 18F-FDG PET/CT in the evaluation of adrenal masses. J Nucl Med 2006; 47: 32-7.

37. Bury T, Barreto A, Daenen F et al. Fluorine-18 deoxyglucose positron emisión tomography for the detection of bone metastases in patients with non-small cell lung cancer. Eur J Med Nucl Med 1998; 16: 3375-3379.

38. Cook GJ, Houston S, Rubens R, et al. Detection of bone metastaes in breast cancer by 18FDG PET: Differing metabolic activity in osteoblastic and osteolityc lesions. J Clin Oncol 1998; 16: 3375-3379.

39. Meyer M, Gast T, Raja S, et al. Increased F-18-FDG accumulation in an acute fracture. Clin Nucl Med 1994; 19: 1314.

40. Simó M, Cirera L, García-Garzón JR, et al. Impacto clínico de la PET con 18FDG en la selección de la terapia de los pacientes oncológicos. Oncología 2006; 29(4): 150-157.

41. Hicks RJ, Kalff V, MacManus MP, et al. (18)F-FDG PET provides high-impact and powerful prognostic stratification in staging newly diagnosed non-small cell lung cancer. J Nucl Med 2001; 42: 1596-604.

42. Schmucking M, Baum RP, Griesinger F, et al. Molecular whole cancer staging using positron emission tomography: consequences for therapeuticmanagement and metabolic radiation treatment planning. Recent Results Cancer Res 2003; 162: 195-202.

43. Mankoff DA, Bellon JR. Positron-emission tomographic imaging of cancer: Glucose metabolism and beyond. Sem Radiat Oncol 2001; 11: 16-27.

44. Stroobants S, Verschakelen J, Vansteenkiste J. value of FDG-PET in the management of non-small cell lung cancer. Eur J Radiol 2003; 45:49-59.

45. Veselle H, Schmidt RA, Pugley JM, et al. Lung cancer proliferation correlates with [F-18] fluorodeoxyglucose uptake by positron emission tomography. Clin Cancer Res 2000; 6: 3837-3844.

46. Bos R, van der Hoeven JJ, van Der Wall E, et al. Biologic correlates of 18flurodeoxyglucose uptake in human breast 
cancer mesasured by positron emission tomography. J Clin Oncol 2002; 20: 379-387.

47. Duhaylongsod FG, Lowe, Patz EF, et al. Lung tumor growth correlates with glucose metabolism measured by fluride-18-fluodeoxygluose positron emission tomography. Ann Thorac Surg 1995; 60: 1348-1352.

48. Ahuja V, Coleman RE, Herndon J, Patz EF. The prognostic significance of fluorodeoxyglucosa positron emission tomography imaging for patients with non-small-cell-lung cancer.Cancer 1998; 83: 918-24.

49. Vansteenkiste JF; Stroobants SG, De Leyn PR, et al. Prognostic importance of the standardized uptake value on FDG-PET scan in non-small cell lung cancer: an analysis of 125 cases. J Clin Oncol 1999; 17: 3201-06.

50. Dhital K, Saunders CA, Seed PT, et al. [18F] Fluorodeoxyglucose positron emission tomography and its prognostic value in lung cancer. Eur J Cardiothorac Surg 2000; 18:425-28.

51. Higashi K, Ueda Y, Arisaka Y, et al. 18F-FDG upptake as a biologic prognostic for factor recureence in patients with surgically resected non-small cell lung cancer. J Nucl Med 2002; 43:39-45.

52. Jeong HJ, Min JJ, Park JM, et al. Determination of the prognostic value of [(18) F]fluorodeoxyglucose uptake by using positron emission tomography in patients with nonsmall cell lung cancer. Nucl Med Commun 2002; 23:86570.

53. Borst GR, Belderbos JSA, Boellaard R, et al. Captación de FDG estandarizada: un factor pronóstico en el cáncer de pulmón no microcítico inoperable. Eur J Cancer (ed. española) 2005; 41: 1533-1541.

54. Sasaki R, Komaki R, Macapinlac H, et al. [18F] Fluorodeoxyglucose uptake by positron emission tomography predicts outcome of non-small-cell lung cancer. J Clin Oncol 2005; 23: 1136-1143.

55. Weber WA. Use of PET for monitoring cancer therapy and for predicting outcome. J Nucl Med 2005; 49: 983-995.

56. Downey RJ, Akhurts T, Gonen M, et al. Preoperative F18 fluorodeoxyglucose-positron emission tomography maximal standardized uptake value predicts survival after lung cancer resection. J Clin Oncol 2004; 22: 32553260 .

57. Stahl A, Ott K, Schwaiger M, Weber WA. Comparison of different SUV-based methods for monitoring cytotoxic therapy with FDG PET. Eur J Nucl Med Mol Imaging 2004; 31: 1471-1478.

58. Junker K, Langner K, Klinke F, et al. Grading of tumor regression in non-small-cell lung cancer: Morphology and prognosis. Chest 2001; 120: 1584-1591.

59. Yamane T, Daimaru O, Ito S, Yoshiya K, Nagata T, Uchida H. Decreased 18F-FDG uptake 1 day after initiation of chemotherapy for malignant lymphomas. J Nucl Med 2004; 45: 1838-1842.

60. Saepen K, S, Dupont P, et al. Prognostic value of positron emission tomography (PET) with fluorine-18 fluorodeoxyglucose ([18]FDG) after first-line chemotherapy in non-
Hodgkin's lymphoma: Is [18]FDG-PET a valid alternative to conventional diagnosis methods? J Clin Oncol 2001; 19:414-419.

61. Flamen P, Van Custem E, Lerut AS, et al. Positron emisión tomography for assessment of the response to induction chemotherapy in locally advanced esophageal cancer. Ann Oncol 2002; 13: 361-368.

62. Wieder HA, Brucher BL, Zimmermann F, et al. Time course of tumor etabolic activity during chemoradiotherapy of esophageal squamous cell carcinoma and response to treatment. J Clin Oncol 2004; 22: 900-908.

63. Ott K, Fink U, Becker K, et al. Prediction of response to preoperative chemotherapy in gastric carcinoma by metabolic imaging: results of a prospective trial. J Clin Oncol 2003; 21: 4604-4610.

64. Schulte M, Brecht-Krauss D, Werner M, et al. Evaluation of neoadjuvant therapy response of osteogenic sarcoma using FDG PET. J Nucl Med 1999; 40: 1637-1643.

65. Su H, Bodenstein C, Dumont RA, et al. Monitoring tumor glucose utilization by positron emisión tomography for the prediction of treatment response to epidermal growth factor receptor kinase inhibitors. Clin Cancer Res 2006; 12: 5659-5667.

66. Ichiya Y, Kuwabara Y, Sasaki M et al. A clinical evaluation of FDG-PET to assess the response in radiation therapy for bronchogenic carcinoma. Ann Nucl Med 1996; 10: 193-200.

67. Choi NC, Fischman AJ, Niemierko A, et al. Dose-response relationship between probability of pathologic tumor control and glucose metabolic rate measured with FDG PET after preoperative chemoradiotherapy in locally advanced non-small cell lung cancer. Int J Radiat Oncol Biol Phys 2002; 54:1024-35.

68. MacManus MP, Hicks RJ, MatthewsJP, eta 1. Positron emission tomography is superior to computed tomography scanning for response-assesment after radical radiotherapy or chemoradiotherapy in patients with non-small cell lung cancer. J Clin Oncol 2003; 21: 1285-92.

69. Ryu JS, Choi NC, Fischman AJ, Lynch T, Mathisen DJ. FDG-PET in staging and restaging non-small cell lung cancer after neoadjuvant chemoradiotherapy: correlation with histopathology. Lung Cancer 2002; 35: 179-187.

70. Webwer WA, Petersen V, Schmidt B, et al. Positron emission tomography in non-small cell lung cancer: prediction of response to chemotherapy by quantitative assessment of glucose use. J Clin Oncol 2003; 21: 2651-92.

71. Hoeskstra CJ; Stroobants SG, Smit EF, et al. Prognostic relevance of response evaluation using [18F]-2-Fluoro-2deoxi-D-glucose positron emission tomography in patients with locally advanced Non-small-cell lung cancer. J Clin Oncol 2005; 23:8362-8370.

72. Hoekstra CJ, Paglianiti I, Hekstra OS, et al. Monitoring response to thrapy in cancer using [18F]-2-fluoro-2-D-glucose and positron emisión tomography: An overview of different analytical methods. Eur J Nucl Med 2000; 27: 731-743. 
73. Ciernik IF, Dizendorf E, Baumert BG, et al. Radiation treatment planning with an integrated positron emisión and computer tomography /PET/CT). A feasibility study. Int J Rad Oncol Biol phys 2003; 57: 853-63.

74. Van Der Wel A, Nijsten S, Hochstenbag M et al. Increased therapeutic ratio by 18 FDG-PET CT planning in patients with clinical CT stage N2-N3 M0 non-small-cell lung cancer: A modeling study. Int J Radiation Oncology Biol Phys 2005; 61; 649-655.

75. Mah K, Caldwell C, Ung Y, et al. The impact of (18)FDGPET on target and critical organs in CT-based treatment planning of patients with poorly defined non-small cell liung carcinoma: A prospective study. Int J Radiat Oncol Biol Phys 2002; 52:339-350.

76. Van de Steene J, Linthout N, de Mey J, et al. Definition of gross tumor volume in lung cancer: Interobserver variability. Radiother oncol 2002; 62: 37-49.

77. Steenbakkers RJ, Duppen JC, Fitton I, et al. Observer variation in target volume delineation of lung cancer related to radiation oncologist-computer interaction: A "Big Brother" evaluation. Radiother Oncol 2005; 24: xx-xx

78. Vanuystel L, Vansteenkiste J, Stroobants S, et al. The impact of 18F-fluoro-2-deoxy-D-glucose positron emission tomography (FDG-PET) lymph node staging on the radiation treatment volumes in patients with non-small cell lung cancer. Radiother Oncol 2000; 55: 317-324.

79. Bradley J, Thorsted WL, Sasa Mutic MS, et al. Impact of FDG-PET on radiation therapy volume delineation in nonsmall-cell lung cancer. Int J Radiation Oncology Biol Phys 2004; 59: 78-86.

80. Deniaud-Alexandre E, Touboul E, Lerouge D, et al. Impact of computed tomography and 18F-Deoxyglucose coincidence detection emission tomography image fusion for optimization of conformal radiotherapy in non-smallcell lung cancer. Int J Radiation Oncology Biol Phys 2005; 63: 1432-41.

81. Bruzzi JF, Truong MT, Marom EM, et al. Incidental findings on integrated PET/CT that do not accumulate $18 \mathrm{~F}-$ FDG. AJR 2006; 187: 1116-1123.

82. Aquino SL, Asmuth JC, Alpert NM, Halpen EF, Fischman AJ. Improved radiologic staging of lung cancer with 2-
[18F]-fluoro-2-deoxy-D-glucose-'positron emission tomography and computed tomography registration. J Comput assist Tomogr 2003; 27:479-484.

83. Verschakelen JA, De Wever W, Bogaert J. Role of computed tomography in lung cancer staging. Curr Opin Pulm Med 2004; 27: 479-484.

84. Cerfolio RJ, Ojha B, Bryant AS, Raghuveer V, Mountz JM, Bartolucci AA: The accuracy of integrated PET-CT compared with dedicated PET alone for the staging of patients with non small cell lung cancer. Ann Thorac Surg 2004; 78: 1017-23.

85. Antoch G, Stattaus J, Nemat AT, et al. Non-small cell lung cancer: ndual-modality PET/CT in preoperative staging. Radiology 2003; 229: 526-533.

86. Vansteenkiste JF, Stroobants SG, De Leyn PR, et al. Leuven Lung Cancer Group. Mediastinal lymph node staging with FDG-PET scan in patients with potentially operable non-small cell lung cancer: a prospective analysis of 50 cases. Chest 1997; 112: 1480-1486.

87. Halpern BS, Schiepers C, Weber WA, et al. Presurgical staging of non-small cell lung cancer: positron emission tomography, integrated positron emission tomography/ CT, and software image fusion. Chest 2005; 128: 228997.

88. Pozo-Rodríguez F, Martín de Nicolás JL, Sánchez-Nistal MA, et al. Accuracy of helical computed tomography and [18F] Fluorodeoxyglucose positron emission tomography for identifying lymph node mediastinal metastases in potencially resectable non-small-cell lung cancer. J Clin Oncol 2005; 23: 8348-8356.

89. Bar-Shalom R, Yefremov N, Guralnik L, et al. Clinical performance of PET/CT in evaluation of cancer: Additional value for diagnostic imaging and patient managememt. J Nucl Med 2003; 44: 1200-1209.

90. Rodríguez Garrido M, Asensio del Barrio C. PET-TAC: Indicaciones, revisión sistemática y meta-análisis. Madrid: AETS-Instituto de Salud Carlos III, Junio 2004.

91. Shim SS, Lee KS, Kim BT, et al. Non-small cell lung cancer: prospective comparison of integrated FDG PET/T and CT alone for preoperative staging. Radiology 2005; 236:1011-9. 